\title{
THE CONTACT GLASS AS AN AID IN CORNEAL PLASTIC SURGERY
}

\author{
BY
}

\author{
LT.-Col. R. E. WRIGHT
}

THE value of the conjunctival flap in dealing with wounds and ulcers of the cornea and limbus, whether perforating and liable to uveal involvement or non-perforating, is generally recognised.

The method has proved of greatest service perhaps in the treatment of ulcers followed by iris incarceration. Descemetocele, or fistula, and wounds, non-operative or operative associated with iris prolapse, $e . g$., prolapse following a cataract operation.

According to the nature of the condition treated the conjunctival flap may be expected to act either temporarily as a protective covering or permanently as a graft. An example of the former is the covering obtained by making a complete circumcorneal incision of the conjunctiva, undermining it, and drawing it over the cornea by a purse-string suture. In vascular conditions of the cornea such a flap may behave as a graft and do much harm by forming an undesired adhesion. In the latter category the apron flap used near the limbus to cover a corneo-scleral defect, e.g., a cataract section with or without prolapse of iris, is usually intended to act as a graft, becoming permanently attached and forming a sound protective covering for the defect.

In perforating lesions towards the pupillary area of the cornea, e.g., a post-ulcerative perforation with iris incarceration and fistula, a permanent covering of the defect by an adherent flap or graft is desirable, but operators with considerable experience are frequently chagrined to find that their best efforts by ordinary methods, such as the pedicle or bridge flap, result in a temporary covering where a permanent one was intended. No doubt such a protective strip of conjunctiva sometimes allows healing to take place under it thus achieving the desired result, but this does not alter the fact that in many cases successful grafting is essential for the best results. With this end in view, towards the end of 1931 I tried several unorthodox methods, some of which at least extend the sphere of usefulness of plastic surgery as applied to the cornea. One of these was adopted in the case of corneal fistula following ulcer in the pupillary area. The patient, a pregnant woman, was expecting delivery in a few weeks, and it was desirable to get a sound closing of the aperture before she left for her confinement. The neighbourhood of the opening was first prepared by carefully destroying the epithelium of the aperture and that for some distance around by a light application of a fine electro-cautery 
point. For this purpose a useful cautery handle is that made for me by Messrs. Down Bros. which resembles a stylographic pen in shape and can be similarly used. The flex leading to a switch operated by knee or foot, takes off from the middle of the handle towards the palm of the hand, thus eliminating drag and rendering delicate touch possible. A pedicle ribbon flap 6-8 $\mathrm{mm}$. wide was raised from the upper bulbar conjunctiva in a transverse direction, one edge of the flap being concentric with the corneo-scleral junction and about $5 \mathrm{~mm}$. from it. To facilitate getting a very thin flap, ballooning the conjunctiva by an injection of novacaine solution is useful. A ribbon flap is thus easily cut with a cataract knife. The defect in the conjunctiva was next closed by a few points of very fine suture. A continuous suture for this purpose would do equally well. The flap was then laid over the cornea and stretched out thin and level over the raw area, its free end being buried in a subconjunctival pocket made at the limbus at a point diametrically opposite its attached end. A fairly flat contact glass was then applied which held the flap stretched in position. It was not removed for seven days but the flap was very carefully inspected through the glass from the second day onwards. On the fourth day it seemed that the flap had failed to take as its end was free inside the concavity of the glass. On removing the glass carefully three days later it was seen that the graft had taken, immediately over the prepared area. The unadherent flap was carefully cut away and although the attachment was of a very delicate nature, the lids were closed without replacing the glass, covered with ointment lint and both eyes bandaged. A good sound graft was assured by the tenth day which enabled the patient to leave hospital with a safe eye and return after her confinement with a good firm scar, for further observation as to optical treatment. A modification of this method in which a free conjunctival ribbon flap was tried did not succeed. The ends of the flap were at first gripped by the contact glass where they crossed the limbus and extended out on the bulbar conjunctiva but they came free before a "take" was effected. The ends of the ribbon would have been more secure had they been buried in subconjunctival pockets at the limbus on either side.

A third procedure gave an excellent result. Instead of using a conjunctival graft a thin epithelial ribbon was taken from the inner side of the arm. This was laid out evenly over the prepared floor of the ulcer, the ends of the ribbon being buried beneath the limbal conjunctiva at either end of the 45 degree diameter. A contact glass was then placed in position. In this case as the edge of the ulcer base was situated within a few $\mathrm{mm}$. of the limbus, not only was the floor of the ulcer cauterized and its edges denuded of epithelium, but a track of cornea leading from this edge to the limbus was similarly denuded. This has been my routine practice 
in any conjunctival flap procedure where the portion of the cornea between the lesion and the limbus may reasonably be sacrificed as it gives the graft a much better chance of vascularisation. Even in defects near the centre it is advisable to raw a narrow track of cornea in the proposed axis of the ribbon flap on one or both sides of the defect.

The epithelial graft placed as above described adhered soundly to the raw area and the glass was removed on the seventh day. It was then found when trimming off the excess of graft that it had also adhered firmly to the episclera in the pockets and this had to be carefully removed. The result was excellent, the small rounded patch of epithelium which covered the old ulcer soon became smooth and moist.

This modification, grafting with skin instead of conjunctiva, has some very obvious advantages and is worthy of consideration and further development. Possibly the idea is not new but as far as could be ascertained at the time, the method adopted was original. The actual technique might be improved so as to avoid adhesion of the buried ends of the ribbon. Instead of a contact glass a small thin paraffin shell adapted in size and shape to the cornea might be used, placing in its concavity an epithelial graft somewhat larger than the defect to be covered, and the whole held in place by means of raising a complete concentric conjunctival flap at the limbus and drawing it together over the shell with a purse-string suture. A detached graft lining a paraffin shell of the same size as an ordinary glass eye is obviously unsuitable for corneal work, but is excellent for defects of the conjunctival fornices, whether mucous membrane or skin be used. In fact paraffin of a suitable melting point is invaluable for the making of shells and forms to carry all manner of grafts in connection with conjunctival plastics.

When experimenting with contact glasses as an aid to grafting, it occured to me that they could also be used in the treatment of corneal ulcers, in order to maintain an antiseptic agent in effective strength in contact with the lesion, e.g., iodoform. This was tried but my experience of the method is insufficient to justify an opinion as to its value. 